\title{
In-operando photoelectron spectroscopy for batteries: Set-up using pristine thin film cathode and first results on $\mathrm{Na}_{x} \mathrm{CoO}_{2}$
}

Cite as: Rev. Sci. Instrum. 89, 073104 (2018); https://doi.org/10.1063/1.5022616

Submitted: 17 January 2018 . Accepted: 06 June 2018 . Published Online: 10 July 2018

Conrad Guhl (D), Philipp Kehne, Qianli Ma, Frank Tietz (D), Lambert Alff, Philipp Komissinskiy, Wolfram Jaegermann, and René Hausbrand

Eiew Online

\section{ARTICLES YOU MAY BE INTERESTED IN}

Development of angle-resolved spectroscopy system of electrons emitted from a surface with negative electron affinity state

Review of Scientific Instruments 89, 073103 (2018); https://doi.org/10.1063/1.5021116

A novel laboratory-based hard X-ray photoelectron spectroscopy system

Review of Scientific Instruments 89, 073105 (2018); https://doi.org/10.1063/1.5039829

Time-resolved orientation detection system with quantum cascade lasers

Review of Scientific Instruments 89, 073101 (2018); https://doi.org/10.1063/1.5028431

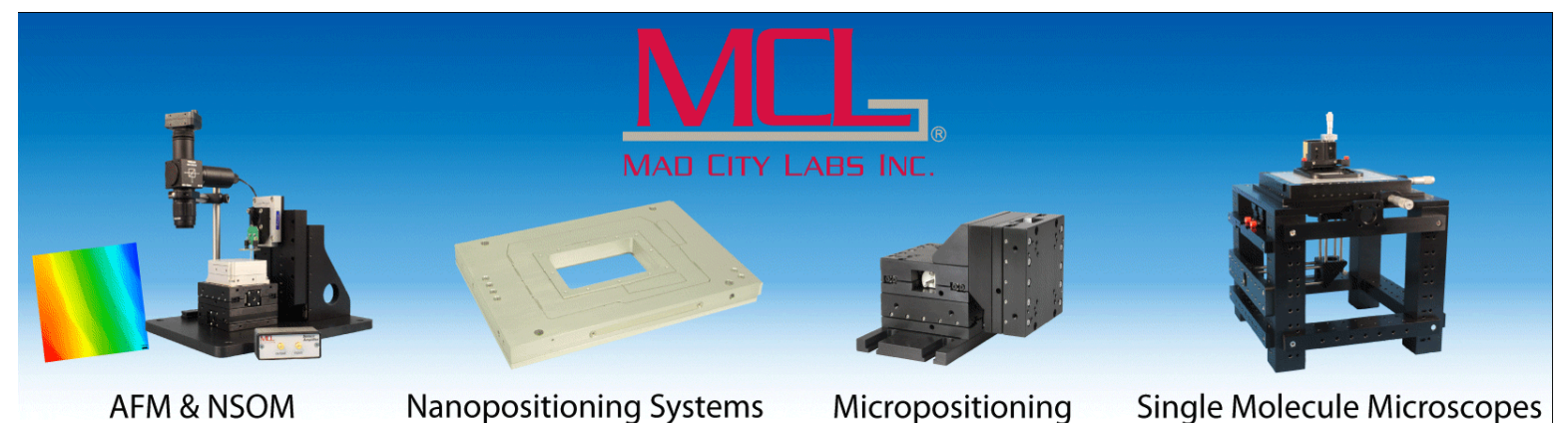




\title{
In-operando photoelectron spectroscopy for batteries: Set-up using pristine thin film cathode and first results on $\mathrm{Na}_{\mathrm{x}} \mathrm{CoO}_{2}$
}

\author{
Conrad Guhl, ${ }^{1}$ Philipp Kehne, ${ }^{2}$ Qianli Ma, ${ }^{3,4}$ Frank Tietz, ${ }^{3,4}$ Lambert Alff, ${ }^{2}$ \\ Philipp Komissinskiy, ${ }^{2}$ Wolfram Jaegermann, ${ }^{1}$ and René Hausbrand ${ }^{1}$ \\ ${ }^{1}$ Institute of Materials Science, Surface Science, TU-Darmstadt, Otto-Berndt-Str. 3, 64287 Darmstadt, Germany \\ ${ }_{2}^{2}$ Institute of Materials Science, Advanced Thin Film Technology, TU-Darmstadt, Alarich-Weiss-Str. 2, \\ 64287 Darmstadt, Germany \\ ${ }^{3}$ Forschungszentrum Jülich GmbH, Institute of Energy and Climate Research, Materials Synthesis and Processing \\ (IEK-1), 52425 Jülich, Germany \\ ${ }^{4}$ Helmholtz-Institute Münster, Forschungszentrum Jülich GmbH, 52425 Jülich, Germany
}

(Received 17 January 2018; accepted 6 June 2018; published online 10 July 2018)

\begin{abstract}
A model all-solid-state battery cell with a thin film $\mathrm{Na}_{\mathrm{x}} \mathrm{CoO}_{2}$ cathode was assembled under ultrahigh vacuum conditions and cycled inside the vacuum chamber, using a dedicated sample holder. We present in-operando x-ray photoelectron spectroscopy measurements of a $\mathrm{Na}_{\mathrm{x}} \mathrm{CoO}_{2}$ cathode at different charging states. During battery operation, the change in sodium content, the change in cobalt oxidation state, and the evolution of the O1s and VB emissions could be monitored. Comparison with a conventional post-mortem analysis technique showed that the new measurement technique produces comparable results regarding the oxidation state of the transition metal, but sodium and oxygen results show differences due to cathode/electrolyte interfacial reactions for conventional analysis. By using surface layer-free samples in the presented techniques, we could circumvent such reactions and obtain reliable spectra for the pure bulk-like active cathode material. Published by AIP Publishing. https://doi.org/10.1063/1.5022616
\end{abstract}

\section{INTRODUCTION}

For state-of-the-art rechargeable batteries, the usual operation procedure is the reversible insertion of alkali metal into a host structure, most commonly a layered transition metal oxide. Insertion takes place separately for alkali ions and electrons via the interfaces between the electrodes and electrolyte and is extended into the bulk of the host material, where most of the alkali is stored. The nature of the insertion reaction of alkali into the host structure is a key issue in terms of the performance of the electrode material, ${ }^{1,2}$ for instance electrode potential and reversible capacity. Both are initially coupled to the electronic structure of the host material and its evolution upon deintercalation in relation to standard changes. ${ }^{3}$ Whilst the ions are intercalated, the corresponding electrons are stored in unoccupied electronic states of the host material. For a detailed understanding of alkali storage in intercalation materials, the representative electronic structure of the bulk of the active material and the changes during operation must be studied.

In the past, the electronic structure of intercalation materials was derived from the state of charge (SOC) vs. voltage curves $^{4-6}$ or experimentally determined using electrodes that were electrochemically deintercalated using a liquid electrolyte and measured post-mortem using photoelectron spectroscopy (PES) with different excitation energies. ${ }^{7-9}$ Due to the high reactivity of alkali metals and the complexity of the battery device, the post-mortem analysis was typically performed on partially charged electrodes after disassembly of the battery inside a glovebox..$^{7,10-15}$ The main focus of the post-mortem studies was to investigate the formation and properties of the surface solid electrolyte interface (SEI) layers which stabilize the electrode-electrolyte interface and are essential for the performance of the battery. However, during PES analysis of the pure active material, the SEI layer, that is originally beneficial for device performance, hinders the access to the bulk electrode material for the highly surface-sensitive photoelectron spectroscopy as contributions from the residuals of the electrolyte and the SEI layer involving changes of electrode surface composition are inevitably present in the recorded spectra. ${ }^{12}$ Thus, for a detailed understanding of the fundamental processes in the cathode material itself, post-mortem PES is of limited use, as the probed interface region may not be representative for the active material itself.

A very few photoemission studies on batteries using a solid state electrolyte and pristine thin film electrodes have been performed in situ so far, none of which were conducted using typical state-of-the-art-like battery materials. ${ }^{16-18}$ Tonti et al. studied a $\mathrm{TiS}_{2}$ cathode in a $\mathrm{TiS}_{2} / \beta^{\prime \prime}-\mathrm{Al}_{2} \mathrm{O}_{3} /$ graphite battery setup, ${ }^{17}$ whilst Crumlin et al. investigated a $\mathrm{Li}^{-} \mathrm{O}_{2}$ battery with x-ray photoelectron spectroscopy (XPS) at ambient pressure. ${ }^{16}$ Only very recently, Wu et al. published a study on $\mathrm{LiCoO}_{2}$ studied in-operando after assembly inside a glovebox. ${ }^{19}$ Due to the high practical relevance of layered metal oxides as cathodes, it is tempting to study them by means of in-operando XPS. Due to the high surface sensitivity of XPS, it is desirable to achieve surface layer-free cathode materials as starting samples by sole ultra-high vacuum (UHV) processing.

Here, we report on the first in-operando XPS study of a UHV processed layered oxide cathode material. In order to validate the technique, an all-solid-state $\mathrm{Na}_{\mathrm{x}} \mathrm{CoO}_{2} / \mathrm{NASICON} / \mathrm{Na}$ 
battery cell was investigated. The electronic structure of $\mathrm{Na}_{\mathrm{x}} \mathrm{CoO}_{2}$ is of great interest due to its impact on cathode potential, which is far from trivial in the $\mathrm{Na}_{\mathrm{x}} \mathrm{CoO}_{2}$ system: Several potential steps can be identified during the reversible intercalation of sodium, ${ }^{2,20}$ indicating an interesting relation between the electronic structure and structural changes.

In our approach, we prepared thin films of the cathode material $\mathrm{Na}_{\mathrm{x}} \mathrm{CoO}_{2}$ by pulsed laser deposition (PLD). To avoid surface contaminations, the complete cell assembly was carried out under UHV conditions. In situ plating was used for the anode side, as it is a common practice in thin-film battery research. ${ }^{21}$ Using this approach, it is possible to obtain spectra free of electrolyte residuals. To demonstrate the differences and similarities to conventional studies, a $\mathrm{Na}_{\mathrm{x}} \mathrm{CoO}_{2}$ cathode was charged in a liquid electrolyte and analyzed by means of post-mortem XPS.

With the analytical technique presented here, it is possible to study various cathode materials during operation, given that they can be deposited as thin films and have sufficient electronic conductivity. Most state-of-the-art cathode materials can be deposited via thin-film technologies in UHV, thus enabling the implementation of the presented experimental route. Progress in terms of solid-state electrolyte development enables a systematic selection of the cathode material and the solid electrolyte; different ionic species are also accessible. Current and future battery concepts can therefore benefit from this experimental technique.

\section{EXPERIMENTAL}

Powders of the NASICON solid-state electrolyte with the composition $\mathrm{Na}_{3.3} \mathrm{Sc}_{0.3} \mathrm{Zr}_{1.7}\left(\mathrm{SiO}_{4}\right)_{2}\left(\mathrm{PO}_{4}\right)$ were prepared using a solution-assisted solid-state reaction method. ${ }^{22}$ $\mathrm{NaNO}_{3}$ (VWR, 99.7\%), $\mathrm{Sc}_{2} \mathrm{O}_{3}$ (Projector $\mathrm{GmbH}, 99.5 \%$ ), $\mathrm{ZrO}\left(\mathrm{NO}_{3}\right)_{2}$ (Aldrich, 99\%), $\mathrm{Si}\left(\mathrm{OCH}_{2} \mathrm{CH}_{3}\right)_{4}$ (Merck, 99\%), and $\mathrm{NH}_{4} \mathrm{H}_{2} \mathrm{PO}_{4}$ (Merck, 99\%) were used as starting materials. The NASICON powder produced was pressed into a pellet under a uniaxial pressure of $75 \mathrm{MPa}$ at room temperature. The pressed pellet was then sintered at $1260^{\circ} \mathrm{C}$ for $5 \mathrm{~h}$ and cut into $5 \times 5 \mathrm{~mm}$ NASICON substrates. The substrates were subsequently polished to a shiny surface for further usage. The polished substrates were washed with deionized water to remove slurry residuals and heat-treated at $1000{ }^{\circ} \mathrm{C}$ for $1 \mathrm{~h}$.

For the growth of $\sim 750 \mathrm{~nm}$-thick dense $\mathrm{Na}_{\mathrm{x}} \mathrm{CoO}_{2}$ cathode layers by PLD, the NASICON substrate, clamped in an Omicron-type sample carrier, was heated up to $500{ }^{\circ} \mathrm{C}$ in an oxygen atmosphere at 0.01 mbar. A $\mathrm{KrF}(248 \mathrm{~nm})$ excimer laser radiation with an energy fluence of $1.2 \mathrm{~J} / \mathrm{cm}^{2}$ and a pulse frequency of $5 \mathrm{~Hz}$ was used to ablate the Na-rich $(\mathrm{Na}: \mathrm{Co} \approx$ 3:1) $\mathrm{NaCoO}_{2}$ pellet. ${ }^{23}$ The thickness of various PLD deposited layers was checked by SEM cross sections and was always $700-800 \mathrm{~nm}$. Based on the electrode thickness and mass, it was possible to calculate the electrode active mass assuming a $\mathrm{Na}_{\mathrm{x}} \mathrm{CoO}_{2}$ density of $4.8 \mathrm{~g} / \mathrm{cm}^{3}$. In the next step, the NASICON solid electrolyte substrate coated with a $\mathrm{Na}_{\mathrm{x}} \mathrm{CoO}_{2}$ cathode layer was transferred in UHV to a sputter deposition chamber. A Pt current collector was sputtered in the Ar plasma

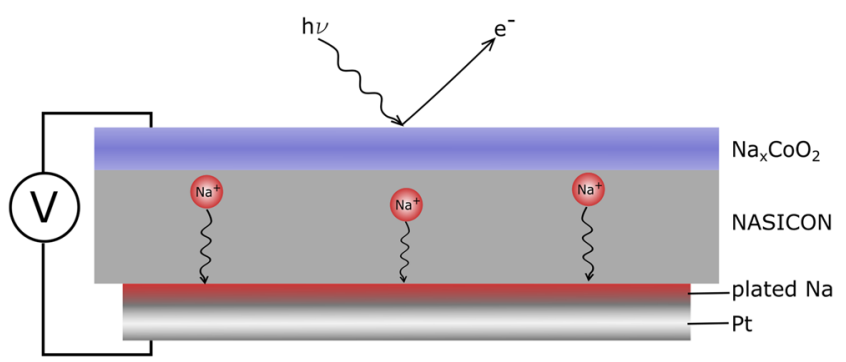

FIG. 1. Schematic cross section of the $\mathrm{Na}_{\mathrm{x}} \mathrm{CoO}_{2} / \mathrm{NASICON/Pt}$ all-solid-state cell in use.

onto the rear side of the NASICON substrate by radio frequency (rf) magnetron sputtering at a power of $20 \mathrm{~W}$, an argon flow of $10 \mathrm{SCCM}$, and a pressure of $3 \times 10^{-3} \mathrm{mbar}$. In order to avoid an electrical contact between the $\mathrm{Pt}$ and $\mathrm{Na}_{\mathrm{x}} \mathrm{CoO}_{2}$ layers, the Pt layer was deposited using a shadow mask to restrict the coating to the middle part of the NASICON substrate. As an external current is applied, $\mathrm{Na}^{+}$migrates through the solid electrolyte and plates a metallic sodium anode at the platinum. A schematic cross section of the cell is shown in Fig. 1.

In order to perform in-operando PES measurements, the Omicron-type sample carrier with the $\mathrm{Na}_{\mathrm{x}} \mathrm{CoO}_{2} / \mathrm{NaSICON} / \mathrm{Pt}$ battery was inserted into a specially designed adapter inside the UHV system. The adapter consists of an insulating PTFE Omicron-type stage mounted onto a Physical Electronics (PHI) type sample holder with electronic insulation between the inner and outer parts (see Fig. 2). The electrodes of the $\mathrm{Na}_{\mathrm{x}} \mathrm{CoO}_{2} / \mathrm{NaSICON} / \mathrm{Pt}$ battery mounted in the adapter were connected from top and bottom by metal springs to the inner and outer sides of the PHI-type sample holder. Within a Physical Electronics VersaProbe XPS stage, a current was applied between the metal springs touching the outer rim of the sample holder and a copper socket contacting the middle pin of the sample holder. In our adapter setup, this resulted in a connection of the top and bottom electrodes of our battery via the spring contacts. The current between the top and bottom springs was applied by using an external Gamry Reference 600 potentiostat, which therefore operated the battery. Since the layered cobalt oxide material studied here is a good electronic conductor, it was sufficient to contact the cathode at only one spot. For adaption of the experimental technique to electrode materials with poor conductivity, a better top contact

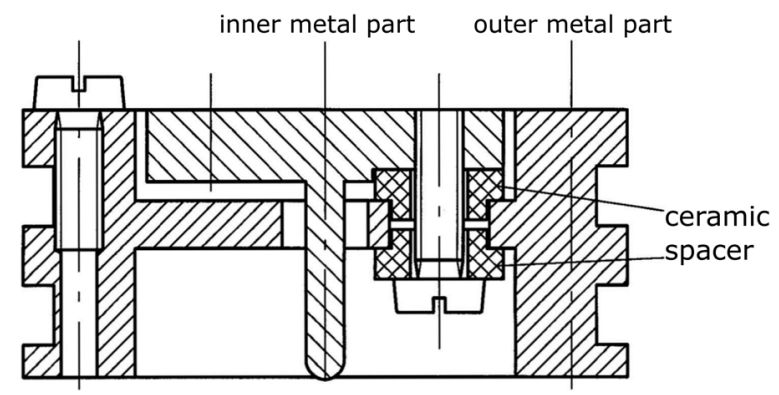

FIG. 2. Cross section of the PHI-type sample holder with insulation between the inner and outer parts. 
(a)

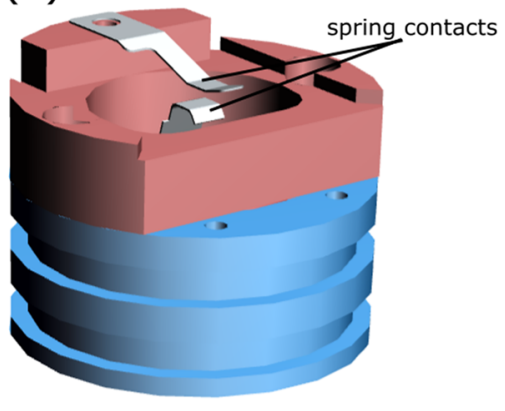

(b)

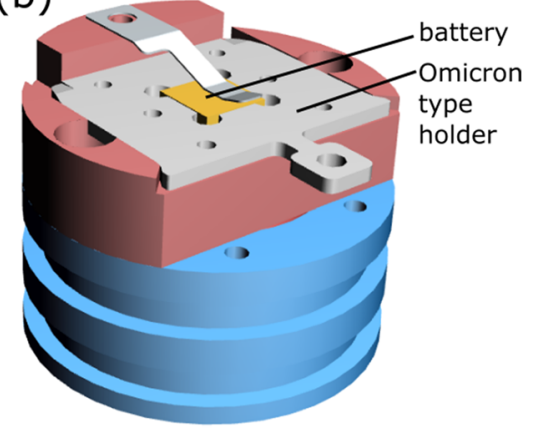

FIG. 3. PHI sample holder (blue) with the mounted Omicron-type stage (red) and metal spring contacts: (a) without and (b) with the Omicron-type sample carrier installed with an all-solid-state cell (as shown in Fig. 1). might be necessary, e.g. a current collector film with a small measurement window. In any case, a part of the surface of the $\mathrm{Na}_{\mathrm{x}} \mathrm{CoO}_{2}$ (in general: electrode) film is not shadowed by the top spring contact (optional: current collector) and can be used to perform XPS measurements during battery operation. The setup with and without Omicron-type sample holder is shown in Fig. 3.

The XPS measurements were performed with a monochromatic Al- $\mathrm{K}_{\alpha}$ source (spot diameter $200 \mu \mathrm{m}$ ) at an angle of $45^{\circ}$ using a fixed analyzer transition mode with a pass energy of $23.5 \mathrm{eV}$ (step size $0.1 \mathrm{eV}$ ). In principle, the experimental setup described allows measurements of the XPS spectra during battery operation. However, as the cell potential can vary significantly during the typical acquisition time of high-resolution spectra $(\sim 2 \mathrm{~h})$, the charging of the battery was interrupted for the XPS measurements. The O1s spectra are superimposed with the Na KLL Auger emission due to the use of an Al-K $\mathrm{K}_{\alpha}$ source. Therefore the spectra were corrected by subtraction of the Na KLL Auger emission based on the Na1s emission intensity.

The results of the in-operando XPS measurements were compared with the data obtained from the conventional postmortem XPS analysis. For the latter, a $\mathrm{Na}_{x} \mathrm{CoO}_{2}$ film was deposited onto a metallic titanium foil (Goodfellow, 99.6+\%) and cycled in a Swagelok-type cell with the metallic sodium anode and liquid electrolyte $\left(1 \mathrm{M} \mathrm{NaClO}_{4}\right.$ in a $45: 45: 10$ $\mathrm{EC} / \mathrm{PC} / \mathrm{DMC}$ mixture). For the post-mortem XPS analysis, the cell was charged to a potential of $3.25 \mathrm{~V}$ and disassembled in an argon-filled glovebox $\left(\mathrm{O}_{2}, \mathrm{H}_{2} \mathrm{O}<0.1 \mathrm{ppm}\right)$. The cathode was washed with DMC and transferred into the UHV system for XPS analysis. To prevent air contact during transport, an Ar-filled transfer chamber was used.

\section{RESULTS AND DISCUSSION}

\section{Electrochemical data}

After assembly of the $\mathrm{Na}_{\mathrm{x}} \mathrm{CoO}_{2} / \mathrm{NaSICON} / \mathrm{Pt}$ cell within the UHV system and transfer to the XPS chamber, the battery was charged in three successive steps to $3.8 \mathrm{~V}$ with a constant current of $500 \mathrm{nA}$. Once a current is applied and sodium is plated at the Pt/NASICON interface, the voltage reaches a typical value for a partially desodiated PLD $\mathrm{Na}_{\mathrm{x}} \mathrm{CoO}_{2}$ film $(\mathrm{x} \sim 0.72)$ vs. sodium. During charging with a constant current, potential steps were observed that are characteristic for $\mathrm{Na}_{\mathrm{x}} \mathrm{CoO}_{2}$ cathode materials ${ }^{20}$ (see Fig. 4).

Following the charge of the battery to $3.8 \mathrm{~V}$ in three successive steps, an accelerated discharge was conducted. Due to the permeability of platinum for sodium, the cell loses sodium, thus resulting in a Coulombic efficiency of $\sim 50 \%$.

\section{Photoemission spectra}

\section{In-operando XPS}

$\mathrm{Na}_{\mathrm{x}} \mathrm{CoO}_{2}$ cathode photoemission spectra of the battery are given in Fig. 5 for different open circuit potentials. Based on the cathode stoichiometry as measured by XPS, a corresponding $\mathrm{Na} / \mathrm{Co}$ ratio ( $\mathrm{x}$ ) is given. For the freshly assembled state, a clear $\mathrm{Na}_{\mathrm{x}} \mathrm{CoO}_{2}$ signature is visible, showing cobalt in

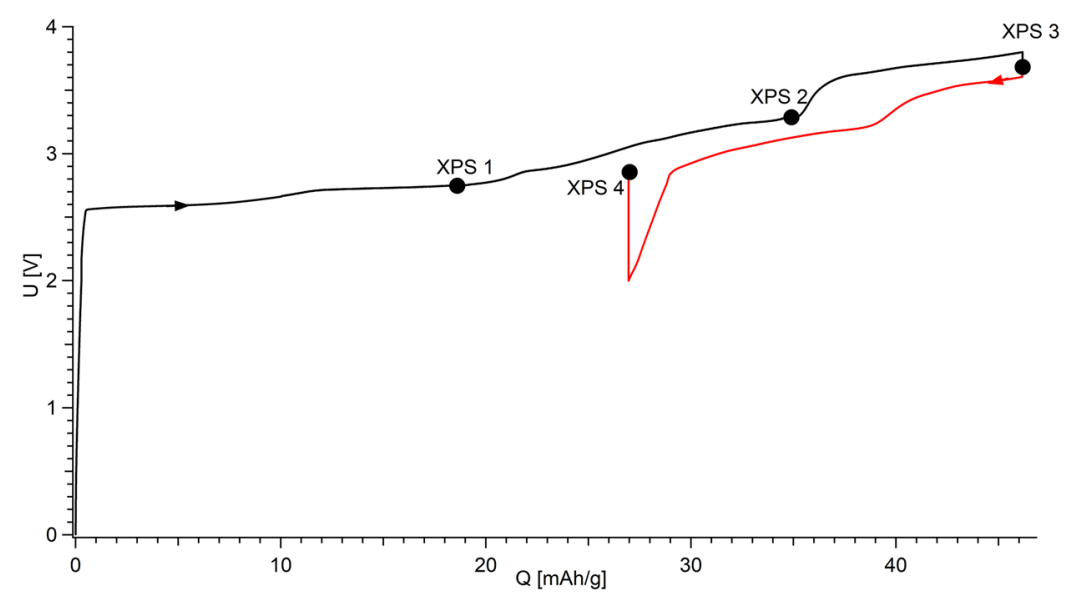

FIG. 4. Charge (black) and discharge (red) curve for the battery during operation inside the XPS chamber. XPS measurement points are marked in the charging curve. Voltage steps characteristic for $\mathrm{Na}_{\mathrm{x}} \mathrm{CoO}_{2}$ were observed, for example after XPS measurement 2. 

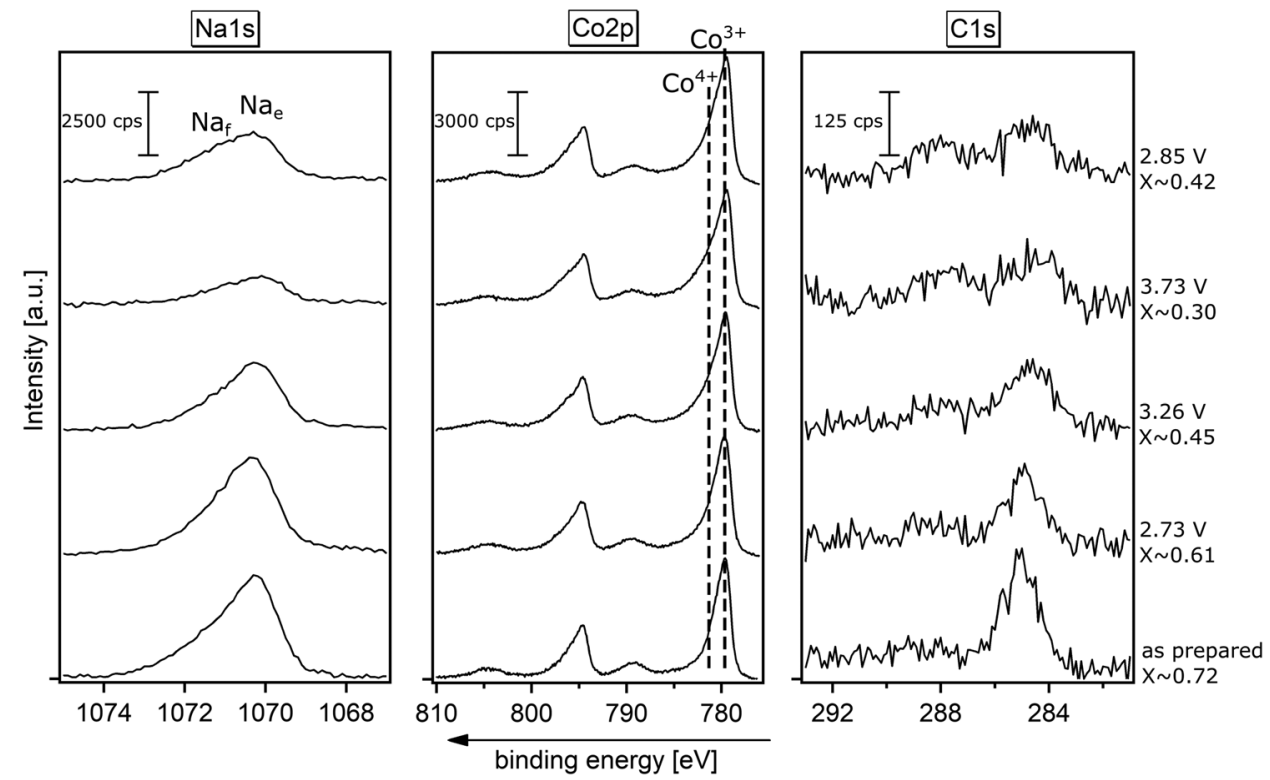

FIG. 5. Na1s, Co2p, and C1s core-level emission lines of the $\mathrm{Na}_{\mathrm{x}} \mathrm{CoO}_{2}$ cathode at different battery potentials during the experiment. The first set of spectra "as prepared" denotes the cathode before any electrochemical treatment, thus without reliable anode and cell potential. The corresponding $\mathrm{Na} / \mathrm{Co}$ ratios are based on XPS stoichiometry measurements. For discussion of spectral assignments: see text.

a mixed valence state $\left(\mathrm{Co}^{3+/ 4+}\right)$ and an asymmetric sodium emission. Slight surface contamination by adsorbed hydrocarbons from the residual gas is evident from the detected $\mathrm{C} 1 \mathrm{~s}$ emission line of the adventitious carbon at $284.8 \mathrm{eV}$. As the battery is charged and the electrode potential becomes more positive, a clear change in the XPS signature can be observed. As expected, the intensity of the sodium emission line decreases and an increase of the $\mathrm{Co}^{4+}$ component in the Co2p core-level signature can be observed. These observations reveal that the entire thin-film cathode can be electrochemically accessed and in-operando XPS can be utilized to analyze the cathode material. Figure 6 shows the photoelectron intensity of the Na1s and $\mathrm{C} 1 \mathrm{~s}$ emission lines. A clear decrease of the sodium line intensity can be seen during charging of the battery, whereas the $\mathrm{C} 1 \mathrm{~s}$ intensity remains weak for all measurements.

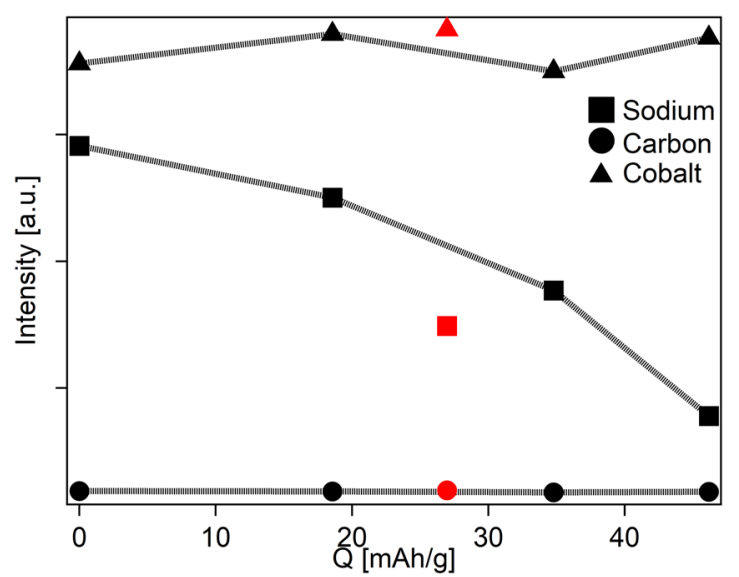

FIG. 6. Sodium, cobalt, and carbon photoelectron intensity as a function of the battery charge; lines between points provide visual guidance. A clear decrease in intensity can be seen for the sodium signal, whereas the carbon and cobalt intensity remains constant. Red symbols are values from the analysis of the discharged cathode.

\section{Na1s}

Sodium exhibits an asymmetric emission for the freshly assembled battery. This shape has been reported for $\mathrm{Na}_{\mathrm{x}} \mathrm{CoO}_{2}$ in the literature and is described as resulting from the different crystallographic positions for sodium inside the compound. $\mathrm{Na}_{\mathrm{f}}$ (face sharing) denotes a sodium ion located directly between two $\mathrm{CoO}_{6}$ octahedra, whereas the $\mathrm{Na}_{\mathrm{e}}$ (edge sharing) site is connected to six $\mathrm{CoO}_{6}$ octahedra. $^{2}$ The $\mathrm{Na}_{\mathrm{f}}$ site is reported to be slightly higher in binding energy. ${ }^{24-26} \mathrm{In}$ the charged state, the lower intensity of the Na1s emission shows that $\mathrm{Na}$ is removed from the $\mathrm{Na}_{\mathrm{x}} \mathrm{CoO}_{2}$ cathode material.

\section{Co2p}

The Co2p spectra consist of four clearly distinguishable components: Co2 $\mathrm{p}_{3 / 2}$ and $\mathrm{Co} 2 \mathrm{p}_{1 / 2}$ as well as two shakeup satellites. In the $\mathrm{Co} 2 \mathrm{p}_{3 / 2}$ emission, the main emission at $779.7 \mathrm{eV}$ is visible from the freshly prepared $\mathrm{Na}_{\mathrm{x}} \mathrm{CoO}_{2}$. Such binding energy is typical for layered cobalt oxide cathode materials of the predominant $\mathrm{Co}^{3+}$ oxidation state. ${ }^{10,27} \mathrm{~A}$ shoulder is present at around $1 \mathrm{eV}$ higher binding energies, thus revealing the presence of $\mathrm{Co}^{4+} \cdot{ }^{28-30}$ Furthermore, a satellite emission positioned at a $9.7 \mathrm{eV}$ higher binding energy than the main emission is also usually attributed to the $\mathrm{Co}^{3+}$ oxidation state. ${ }^{28,31,32}$

The presence of $\mathrm{Co}^{4+}$ is caused by the slightly desodiated starting stoichiometry of the $\mathrm{Na}_{\mathrm{x}} \mathrm{CoO}_{2}$, which agrees with the electrochemical data, with the first charge starting at a potential of $\sim 2.56 \mathrm{~V}$ vs. Na. Upon charging, an increase of the high energy shoulder is observed. This behavior is caused by the increase of the $\mathrm{Co}^{4+}$ component due to the desodiation of the cathode material. The spectral signatures for the as-grown cathode material and for the highly desodiated $\mathrm{Na}_{\mathrm{x}} \mathrm{CoO}_{2}$ at the maximum cell voltage of $3.8 \mathrm{~V}$ are shown in Fig. 7. The data are in good agreement with the expected spectral feature for $\mathrm{Na}_{\mathrm{x}} \mathrm{CoO}_{2}$, with $\mathrm{x}$ ranging from 0.72 (initial) to 0.3 (desodiated). 
(a)

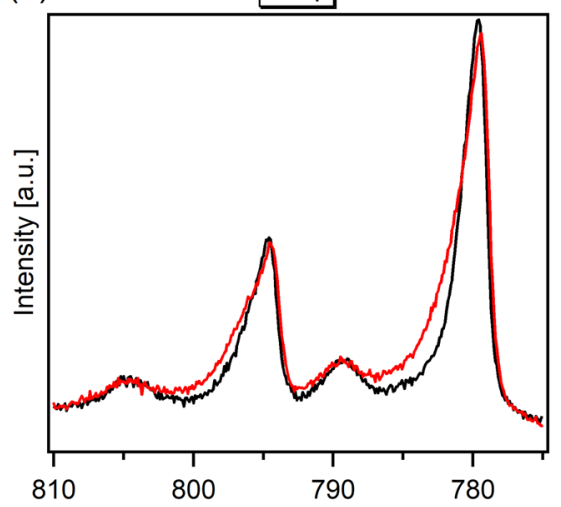

(b)

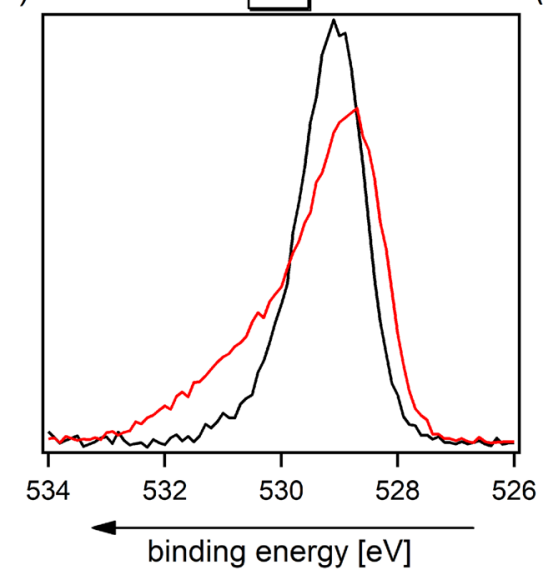

(c)

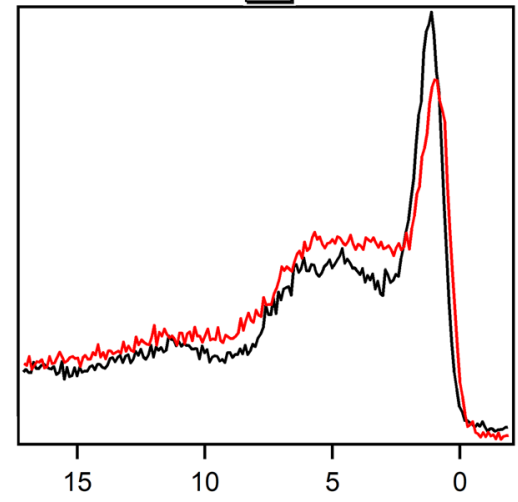

FIG. 7. Comparison of XPS spectra for uncharged (black) and charged (red) $\mathrm{Na}_{\mathrm{x}} \mathrm{CoO}_{2}$ cathode. (a) Co2p core level, (b) O1s core level, and (c) valence band spectra.

\section{c1s}

Minor carbon contaminations were detected on top of the $\mathrm{Na}_{\mathrm{x}} \mathrm{CoO}_{2}$ film due to the high surface sensitivity of XPS. In the initial state, adventitious carbon is present at $284.8 \mathrm{eV}$. This C-contamination is commonly observed in XPS measurements and consists of polymeric hydrocarbon species from residual gas in a vacuum chamber. ${ }^{33}$ As the battery is charged, the adventitious carbon component decreases and a component grows at $\sim 288 \mathrm{eV}$. This behavior reveals a reaction of the surface contaminations, such as an oxidation by the residual gases in the UHV chamber. Based on experience of our group, we presume that this reaction may be catalytically induced by the presence of $\mathrm{Co}^{4+}$ which tends to form free active oxygen species.

Due to the constantly low amount of carbon, we conclude that adsorption or reaction with residual gas, albeit detectable, does not play a major role in our experiments. Such reactions were observed to a much higher extend by the authors using model cells assembled within a glovebox and transferred to an UHV system. ${ }^{18,34}$

\section{1s}

Due to the absence of SEI residuals, it is possible to analyze the $\mathrm{O} 1 \mathrm{~s}$ emission of $\mathrm{Na}_{\mathrm{x}} \mathrm{CoO}_{2}$ in the charged state. Figure 7(b) shows the detailed spectra for a high and low degree of deintercalation, i.e., for low and high battery potential. A shift to lower binding energies can be observed as for the other spectra due to the Fermi level shift during battery charging. Additionally a pronounced shoulder to higher binding energies is visible for the charged state. In principle, this feature could be related to surface layers containing carbon-oxygen species and sodium oxide. However, due to the permanent handling of the sample in UHV, this is not the case here: For a significant contribution of carbon-oxygen species to the O1s spectrum, the amount of surface carbon needs to be higher. In the case of sodium oxide formation, a new high binding energy component in the Na1s emission around $1072.5 \mathrm{eV}$ should be observed but is absent. ${ }^{35}$ Hence, we interpret this spectral feature as the result of an oxygen oxidation process. A detailed discussion of this process will be presented in a subsequent paper. ${ }^{36}$

\section{VB}

In Fig. 7(c), the valence band spectra for the $\mathrm{Na}_{\mathrm{x}} \mathrm{CoO}_{2}$ cathode material in the freshly deposited and deintercalated state can be seen. A clear decrease in emission intensity for the states near the Fermi level can be seen. This decrease corresponds to a depopulation of the Co3d states during battery operation. A detailed investigation of the cathode material electronic structure will be given in a subsequent paper. ${ }^{36}$

\section{Post-mortem XPS analysis}

For the analysis of partially deintercalated $\mathrm{Na}_{\mathrm{x}} \mathrm{CoO}_{2}$ by an ex situ post-mortem XPS technique, different results can be acquired. Due to the presence of SEI residuals, an unambiguous investigation of the pure cathode active material is difficult here. Although such SEI residuals are nevertheless highly interesting and in the focus of numerous investigations, ${ }^{15,37,38}$ such an analysis, however, is not the subject of this paper.

The ex situ post-mortem XPS analysis of the battery with a $\mathrm{Na}_{\mathrm{x}} \mathrm{CoO}_{2}$ thin-film cathode, metallic $\mathrm{Na}$ anode, and liquid electrolyte reveals a much higher amount of carbon on the $\mathrm{Na}_{\mathrm{x}} \mathrm{CoO}_{2}$ cathode layer (Fig. 8). Traces of chlorine originating from $\mathrm{NaClO}_{4}$ in the liquid electrolyte $(1 \mathrm{M} \mathrm{NaClO} 4$ in a 45:45:10 EC/PC/DMC mixture) were also detected. In the Co2p signal, a weaker signal can be seen for the post-mortem sample, although the spectral shape is almost the same. For Na1s, O1s, and C1s emissions, strong contributions of the SEI layer to the spectra are detected. While this spectral information is highly interesting with respect to SEI formation, it masks the bulk features of the cathode active material. A reliable analysis of the oxygen and sodium XPS signal from pure $\mathrm{Na}_{\mathrm{x}} \mathrm{CoO}_{2}$ becomes possible with this technique and will be presented in a subsequent paper. ${ }^{36}$ 


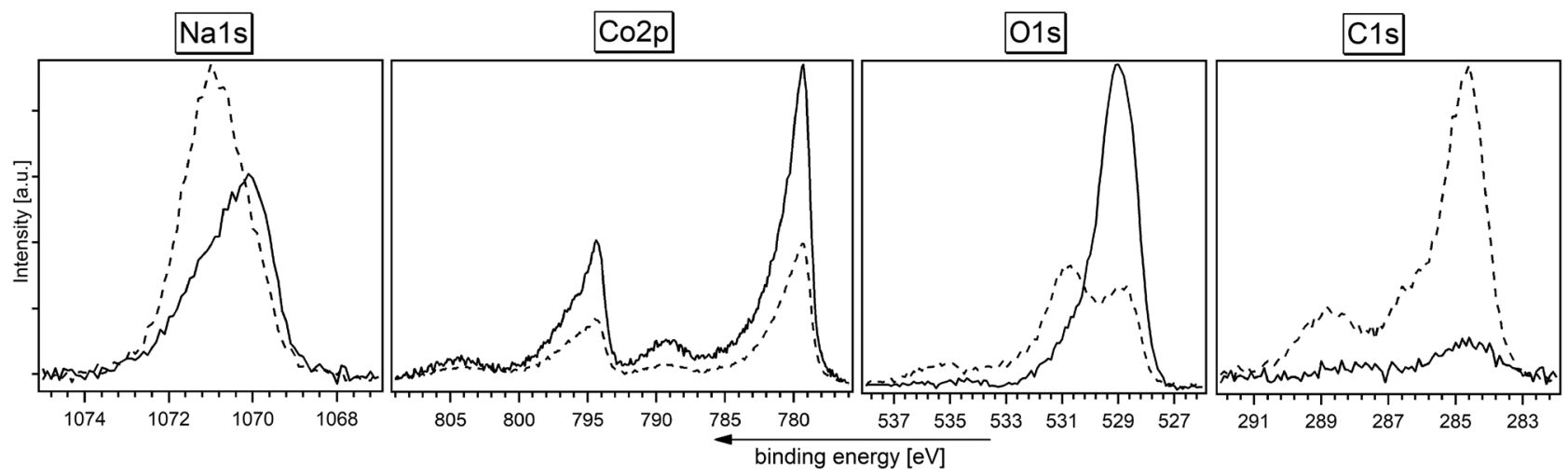

FIG. 8. From left to right: $\mathrm{Na} 1 \mathrm{~s}, \mathrm{Co} 2 \mathrm{p}, \mathrm{O} 1 \mathrm{~s}$, and $\mathrm{C} 1 \mathrm{~s}$ emissions of $\mathrm{Na}_{\mathrm{x}} \mathrm{CoO}_{2}$ cathodes in the charged state at $3.25 \mathrm{~V}$. The spectra with the continuous line were recorded by means of in-operando XPS. The spectra with the dashed line were measured post-mortem on a cathode polarized in a liquid electrolyte after washing with DMC and long-term degassing in the UHV system.

\section{CONCLUSIONS}

A model solid-state battery setup with a thin film layered cathode was successfully used to analyze cathode materials during battery operation in UHV. Using this in-operando XPS method, it is possible to study the layered oxide cathode material in different charging states in the absence of a significant amount of surface layers, resulting in spectral features dominated by the bulk-like material. In the Co2p core-level emission line, the change in the oxidation state of the cobalt cation can clearly be seen by a growth of the spectral component assigned to $\mathrm{Co}^{4+}$.

The change in cobalt oxidation state is comparable to the well-established state-of-the-art post-mortem XPS technique. In contrast to post-mortem analysis, the presented setup results in the surface layer-free battery cathode material. This prevents SEI studies but allows for a more detailed investigation of the changes induced in the pure active material, e.g., the O1s peak. Future work will employ this technique for analysis of the $\mathrm{O} 1 \mathrm{~s}$ core level and valence band spectra as well as the work function measurements without major uncertainties due to previously inevitable electrolyte residuals. This detailed analysis will be presented in a subsequent paper. ${ }^{36}$

We are convinced that the investigation of the chemical behavior and electronic structure of cathodes by means of in-operando PES will deepen our understanding of battery materials in the future as electrons as well as alkali ions are exchanged in de/intercalation cycles. In particular, the important change in the electronic structure of electrode materials in use can be addressed in greater detail using this technique, especially by performing such experiments at synchrotron sources. Benefits are expected to be gained from in-operando XPS studies for the specific material development of future batteries.

\section{ACKNOWLEDGMENTS}

This research project was funded by the DFG (Nos. HA 6128/2-1 and KO 4093/2-1). Additionally, the authors would like to thank BMBF (No. 03KP801) for funding the UHV system DAISY-BAT.
${ }^{1}$ J. N. Reimers and J. R. Dahn, "Electrochemical and in situ X-ray diffraction studies of lithium intercalation in $\mathrm{Li}_{\mathrm{X}} \mathrm{CoO}_{2}$," J. Electrochem. Soc. 139, 2091 (1992).

${ }^{2}$ R. Berthelot, D. Carlier, and C. Delmas, "Electrochemical investigation of the $\mathrm{P} 2-\mathrm{Na}_{\mathrm{x}} \mathrm{CoO}_{2}$ phase diagram," Nat. Mater. 10, 74-80 (2010).

${ }^{3}$ J. B. Goodenough and K. S. Park, "The Li-ion rechargeable battery: A perspective,” J. Am. Chem. Soc. 135, 1167-1176 (2013).

${ }^{4}$ J. B. Goodenough and Y. Kim, "Challenges for rechargeable Li batteries $\dagger$," Chem. Mater. 22, 587-603 (2010).

${ }^{5}$ Y. Kim, K.-S. Park, S.-H. Song, J. Han, and J. B. Goodenough, "Access to $\mathrm{M}^{3+} / \mathrm{M}^{2+}$ redox couples in layered LiMS 2 sulfides $(\mathrm{M}=\mathrm{Ti}, \mathrm{V}, \mathrm{Cr})$ as anodes for Li-ion battery," J. Electrochem. Soc. 156, A703-A708 (2009).

${ }^{6}$ J. Molenda, D. Baster, M. U. Gutowska, A. Szewczyk, R. Puzniak, and J. Tobola, "Electronic origin of the step like character of the discharge curve of $\mathrm{Na}_{\mathrm{x}} \mathrm{CoO}_{2}-\mathrm{y}$ cathode," Funct. Mater. Lett. 07, 1440009 (2014).

${ }^{7}$ L. Daheron, R. Dedryvere, H. Martinez, M. Menetrier, C. Denage, C. Delmas, and D. Gonbeau, "Electron transfer mechanisms upon lithium deintercalation from $\mathrm{LiCoO}_{2}$ to $\mathrm{CoO}_{2}$ investigated by XPS," Chem. Mater. 20, 583-590 (2008).

${ }^{8}$ L. Daheron, H. Martinez, R. Dedryvere, I. Baraille, M. Menetrier, C. Denage, C. Delmas, and D. Gonbeau, "Surface properties of $\mathrm{LiCoO}_{2}$ investigated by XPS analyses and theoretical calculations," J. Phys. Chem. C 113, 5843-5852 (2009).

${ }^{9}$ D. Ensling, G. Cherkashinin, S. Schmid, S. Bhuvaneswari, A. Thissen, and W. Jaegermann, "Nonrigid band behavior of the electronic structure of $\mathrm{LiCoO}_{2}$ thin film during electrochemical Li deintercalation," Chem. Mater. 26, 3948-3956 (2014).

${ }^{10}$ J. J. Ding, Y. N. Zhou, Q. Sun, X. Q. Yu, X. Q. Yang, and Z. W. Fu, "Electrochemical properties of $\mathrm{P} 2$-phase $\mathrm{Na}_{0.74} \mathrm{CoO}_{2}$ compounds as cathode material for rechargeable sodium-ion batteries," Electrochim. Acta 87, 388-393 (2013).

${ }^{11}$ S. Oswald, K. Nikolowski, and H. Ehrenberg, "Quasi in situ XPS investigations on intercalation mechanisms in Li-ion battery materials," Anal. Bioanal. Chem. 393, 1871-1877 (2009).

${ }^{12}$ J. C. Dupin, D. Gonbeau, H. Benqlilou-Moudden, P. Vinatier, and A. Levasseur, "XPS analysis of new lithium cobalt oxide thin-films before and after lithium deintercalation," Thin Solid Films 384, 23-32 (2001).

${ }^{13}$ J. C. Dupin, D. Gonbeau, I. Martin-Litas, P. Vinatier, and A. Levasseur, "Lithium intercalation/deintercalation in transition metal oxides investigated by x-ray photoelectron spectroscopy," J. Electron Spectrosc. Relat. Phenom. 120, 55-65 (2001).

${ }^{14}$ Y.-C. Lu, A. N. Mansour, N. Yabuuchi, and Y. Shao-Horn, "Probing the origin of enhanced stability of 'AlPO 4 ' nanoparticle coated $\mathrm{LiCoO}_{2}$ during cycling to high voltages: Combined XRD and XPS studies," Chem. Mater. 21, 4408-4424 (2009).

${ }^{15}$ N. Schulz, R. Hausbrand, C. Wittich, L. Dimesso, and W. Jaegermann, "XPS-surface analysis of SEI layers on Li-ion cathodes: Part II. SEIcomposition and formation inside composite electrodes," J. Electrochem. Soc. 165, A833-A846 (2018).

${ }^{16}$ E. J. Crumlin, H. Bluhm, and Z. Liu, "In situ investigation of electrochemical devices using ambient pressure photoelectron spectroscopy," J. Electron Spectrosc. Relat. Phenom. 190, 84-92 (2013). 
${ }^{17}$ D. Tonti, C. Pettenkofer, and W. Jaegermann, "In situ photoelectron spectroscopy study of a $\mathrm{TiS}_{2}$ thin film cathode in an operating $\mathrm{Na}$ intercalation electrochemical cell," Ionics 6, 196-202 (2000).

${ }^{18}$ C. Y. Tang, R. T. Haasch, and S. J. Dillon, "In situ X-ray photoelectron and Auger electron spectroscopic characterization of reaction mechanisms during Li-ion cycling," Chem. Commun. 52, 13257-13260 (2016).

${ }^{19}$ X. Wu, C. Villevieille, P. Novak, and M. El Kazzi, "Monitoring the chemical and electronic properties of electrolyte-electrode interfaces in all-solid-state batteries using operando x-ray photoelectron spectroscopy," Phys. Chem. Chem. Phys. 20, 11123 (2018).

${ }^{20}$ C. Delmas, J. J. Braconnier, C. Fouassier, and P. Hagenmuller, "Electrochemical intercalation of sodium in $\mathrm{Na}_{\mathrm{x}} \mathrm{CoO}_{2}$ bronzes," Solid State Ionics 3-4, 165-169 (1981).

${ }^{21}$ B. J. Neudecker, N. J. Dudney, and J. B. Bates, “'Lithium-free' thin-film battery with in situ plated Li anode," J. Electrochem. Soc. 147, 517-523 (2000).

${ }^{22}$ Q. Ma, M. Guin, S. Naqash, C.-L. Tsai, F. Tietz, and O. Guillon, "Scandiumsubstituted $\mathrm{Na}_{3} \mathrm{Zr}_{2}\left(\mathrm{SiO}_{4}\right)_{2}\left(\mathrm{PO}_{4}\right)$ prepared by a solution-assisted solid-state reaction method as sodium-ion conductors," Chem. Mater. 28, 4821-4828 (2016).

${ }^{23}$ S. Hildebrandt, P. Komissinskiy, M. Major, W. Donner, and L. Alff, "Epitaxial growth and control of the sodium content in $\mathrm{Na}_{\mathrm{x}} \mathrm{CoO}_{2}$ thin films," Thin Solid Films 545, 291-295 (2013).

${ }^{24}$ T. Kroll, "On the electronic structure of layered sodium cobalt oxides," Ph.D. dissertation (TU-Dresden, 2006)

${ }^{25}$ P. Zhang, R. B. Capaz, M. L. Cohen, and S. G. Louie, "Theory of sodium ordering in $\mathrm{Na}_{\mathrm{x}} \mathrm{CoO}_{2}$," Phys. Rev. B 71, 153102 (2005).

${ }^{26}$ J. Molenda, D. Baster, A. Milewska, K. Świerczek, D. K. Bora, A. Braun, and J. Tobola, "Electronic origin of difference in discharge curve between $\mathrm{Li}_{\mathrm{x}} \mathrm{CoO}_{2}$ and $\mathrm{Na}_{\mathrm{x}} \mathrm{CoO}_{2}$ cathodes," Solid State Ionics 271, 15-27 (2015).

${ }^{27}$ M. Motzko, M. A. Carrillo Solano, W. Jaegermann, and R. Hausbrand, "Photoemission study on the interaction between $\mathrm{LiCoO}_{2}$ thin films and adsorbed water," J. Phys. Chem. C 119, 23407-23412 (2015).
${ }^{28}$ D. Ensling, "Photoelektronenspektroskopische untersuchung der elektronischen struktur dünner lithiumkobaltoxidschichten," Ph.D. dissertation (TU-Darmstadt, 2006).

${ }^{29}$ J. van Elp, J. L. Wieland, H. Eskes, P. Kuiper, G. A. Sawatzky, F. M. F. de Groot, and T. S. Turner, "Electronic structure of CoO, Li-doped CoO, and $\mathrm{LiCoO}_{2}$," Phys. Rev. B 44, 6090-6103 (1991).

${ }^{30}$ M. Miclau, K. Bokinala, and N. Miclau, "Low-temperature hydrothermal synthesis of the three-layered sodium cobaltite $\mathrm{P} 3-\mathrm{Na}_{\mathrm{x}} \mathrm{CoO}_{2}(\mathrm{x} \sim 0.60)$," Mater. Res. Bull. 54, 1-5 (2014).

${ }^{31}$ V. R. Galakhov, V. V. Karelina, D. G. Kellerman, V. S. Gorshkov, N. A. Ovechkina, and M. Neumann, "Electronic structure, $\mathrm{x}$-ray spectra, and magnetic properties of the $\mathrm{LiCoO}_{2}$-delta and $\mathrm{Na}_{\mathrm{x}} \mathrm{CoO}_{2}$ nonstoichiometric oxides," Phys. Solid State 44, 266-273 (2002).

${ }^{32}$ G. Cherkashinin, D. Ensling, and W. Jaegermann, "LiMO $2(\mathrm{M}=\mathrm{Ni}, \mathrm{Co})$ thin film cathode materials: A correlation between the valence state of transition metals and the electrochemical properties," J. Mater. Chem. A 2, 3571 (2014).

${ }^{33}$ T. L. Barr and S. Seal, "Nature of the use of adventitious carbon as a binding energy standard," J. Vac. Sci. Technol., A 13, 1239-1246 (1995).

${ }^{34}$ C. Y. Tang, K. Leung, R. T. Haasch, and S. J. Dillon, "LiMn ${ }_{2} \mathrm{O}_{4}$ surface chemistry evolution during cycling revealed by in situ Auger electron spectroscopy and x-ray photoelectron spectroscopy," ACS Appl. Mater. Interfaces 9, 33968-33978 (2017).

${ }^{35}$ A. Barrie and F. J. Street, "An Auger and x-ray photoelectron spectroscopic study of sodium metal and sodium oxide," J. Electron Spectrosc. Relat. Phenom. 7, 1 (1975).

${ }^{36}$ C. Guhl, P. Kehne, Q. Ma, F. Tietz, L. Alff, P. Komissinskiy, W. Jaegermann, and R. Hausbrand, (unpublished).

${ }^{37}$ K. Edström, T. Gustafsson, and J. O. Thomas, "The cathode-electrolyte interface in the Li-ion battery," Electrochim. Acta 50, 397-403 (2004).

${ }^{38}$ K. Yamamoto, T. Minato, S. Mori, D. Takamatsu, Y. Orikasa, H. Tanida, K. Nakanishi, H. Murayama, T. Masese, T. Mori, H. Arai, Y. Koyama, Z. Ogumi, and Y. Uchimoto, "Improved cyclic performance of lithium-ion batteries: An investigation of cathode/electrolyte interface via in situ totalreflection fluorescence X-ray absorption spectroscopy," J. Phys. Chem. C 118, 9538-9543 (2014). 